\title{
Ultraviolet-light-induced processes in germanium-doped silica
}

\author{
Kristensen, Martin
}

Published in:

Physical Review B

Link to article, DOI:

10.1103/PhysRevB.64.144201

Publication date:

2001

Document Version

Publisher's PDF, also known as Version of record

Link back to DTU Orbit

Citation (APA):

Kristensen, M. (2001). Ultraviolet-light-induced processes in germanium-doped silica. Physical Review $B$, 64(14), 144201. https://doi.org/10.1103/PhysRevB.64.144201

\section{General rights}

Copyright and moral rights for the publications made accessible in the public portal are retained by the authors and/or other copyright owners and it is a condition of accessing publications that users recognise and abide by the legal requirements associated with these rights.

- Users may download and print one copy of any publication from the public portal for the purpose of private study or research.

- You may not further distribute the material or use it for any profit-making activity or commercial gain

- You may freely distribute the URL identifying the publication in the public portal

If you believe that this document breaches copyright please contact us providing details, and we will remove access to the work immediately and investigate your claim. 


\title{
Ultraviolet-light-induced processes in germanium-doped silica
}

\author{
M. Kristensen \\ Research Center COM, DTU, Building 345 west, DK-2800 Kgs. Lyngby, Denmark
}

(Received 8 May 2001; published 17 September 2001)

\begin{abstract}
A model is presented for the interaction of ultraviolet (UV) light with germanium-doped silica glass. It is assumed that germanium sites work as gates for transferring the excitation energy into the silica. In the material the excitation induces forbidden transitions to two different defect states which are responsible for the observed refractive index changes. Activation energies [1.85 $\pm 0.15 \mathrm{eV}$ and $1.91 \pm 0.15 \mathrm{eV}]$ and rates $[(2.7 \pm 1.9)$ $\times 10^{13} \mathrm{~Hz}$ and $\left.(7.2 \pm 4.5) \times 10^{13} \mathrm{~Hz}\right]$ are determined for thermal elimination of these states. Good agreement is found with experimental results and new UV-induced effects are predicted.
\end{abstract}

DOI: 10.1103/PhysRevB.64.144201 PACS number(s): 71.55.-i, 78.20.-e, 81.40.-z, 82.50. $-\mathrm{m}$

\section{INTRODUCTION}

Since the discovery of light-induced refractive index changes in silica glass materials ${ }^{1}$ and the invention of the side-writing method, ${ }^{2}$ UV writing of gratings in optical fibers has become an important technological field of great commercial importance for telecommunications and optical sensors. However, fundamental understanding of the UVinduced processes in glass materials has lagged far behind the technological development. In brief, there are two competing classes of models for UV-induced index changes in germanium-doped silica. Microscopic models are based on the assumption that defects formed in the glass lead to a higher refractive index. ${ }^{3-7}$ A large number of defects have been identified using different spectroscopic methods and theoretical calculations. However, no satisfactory quantitative agreement has been found with experimentally observed index changes. The second class of models is based on macroscopic changes in the glass. ${ }^{8-11}$ In these models it is assumed that the UV light induces compaction or stress changes in the glass, leading to refractive index changes. In some cases good qualitative agreement is found with experimental results, but there seem to be problems establishing a general quantitative agreement for cases with different geometry or different types of germanium-doped silica. In addition, a macroscopic model lacks the ability to explain what happens on the microscopic level and the ability to give predictions about how to improve the base materials. Two of the most important parameters for UV induced index changes are the UV sensitivity and the stability of the induced changes. A large number of publications discuss how to increase the UV sensitivity of glasses, but there is no consensus on what UV-sensitivity really is. The reason is probably that in most cases the amount of UV-induced index change depends on several parameters such as wavelength, power level, fluence and polarization of the UV light, and temperature and previous treatment of the sample with, e.g., hydrogen. The dependences on fluence and loading seem to be the best studied, because of their commercial importance. The index change is not even a simple, linear function of fluence, but rather a complicated curve which does not allow a straightforward definition of the UV sensitivity. Concerning loading there is consensus that treatment of the samples with hydrogen or deuterium increases the UV sensitivity.
The other important aspect of UV-induced processes in glass is the decay of the refractive index changes. As commercial products based on UV writing find increasing application in telecommunications and as optical sensors, accelerated aging tests have been performed to determine their stability. Most of the results are analyzed using a model that assumes the index changes are due to a very broad spectrum of defects with activation energies from $0.5 \mathrm{eV}$ to $3.5 \mathrm{eV} .^{12,13}$ However, to the best of my knowledge no articles discuss whether such a broad smooth spectrum is physically reasonable and only limited work has been done to relate the annealing results to the models for UV-induced processes. This paper is devoted to the development of a model which describes UV writing near $242 \mathrm{~nm}$, the influence of hydrogen loading, and the thermal erasure in germanium-doped silica. In addition the model gives an explanation of many spectroscopic observations during and after UV irradiation.

The model is based on the assumption that germanium sites in the glass, work as gates for the transfer of energy from the light to the glass matrix. When a germanium site has absorbed a photon, the energy may be transferred to other sites in the glass, leading to rearrangement of the local structure. As will become apparent later the energy transfer happens predominantly through dipole-quadrupole transitions, leading to an $r^{-8}$ dependence of the transition probability as a function of the distance $r$ from the germanium site. ${ }^{14,15}$ In addition, some multiphoton processes are possible using the excited germanium-site state as intermediate level. It is assumed that two different metastable defect states are formed in the silica matrix. One of these is possibly identical to the triplet state identified through $a b$ initio calculations by Sulimov et al. ${ }^{16}$ Formation of the first type of defect in the glass increases the refractive index because it is situated higher in the band gap, bringing its fundamental transitions closer to visible wavelengths. On the other hand, formation of other defects with very small overlap integrals reduces the refractive index, since transitions from these states to more normal electronic glass states are forbidden.

Quantitative agreement is found between the model predictions and a large number of experimental results. For nonsensitized glass the model predicts with good accuracy the scaling of the UV-induced index changes with germanium concentration and even the detailed dynamics during writing. Sensitization includes $\mathrm{H}_{2}$ loading, flame brushing, and spe- 


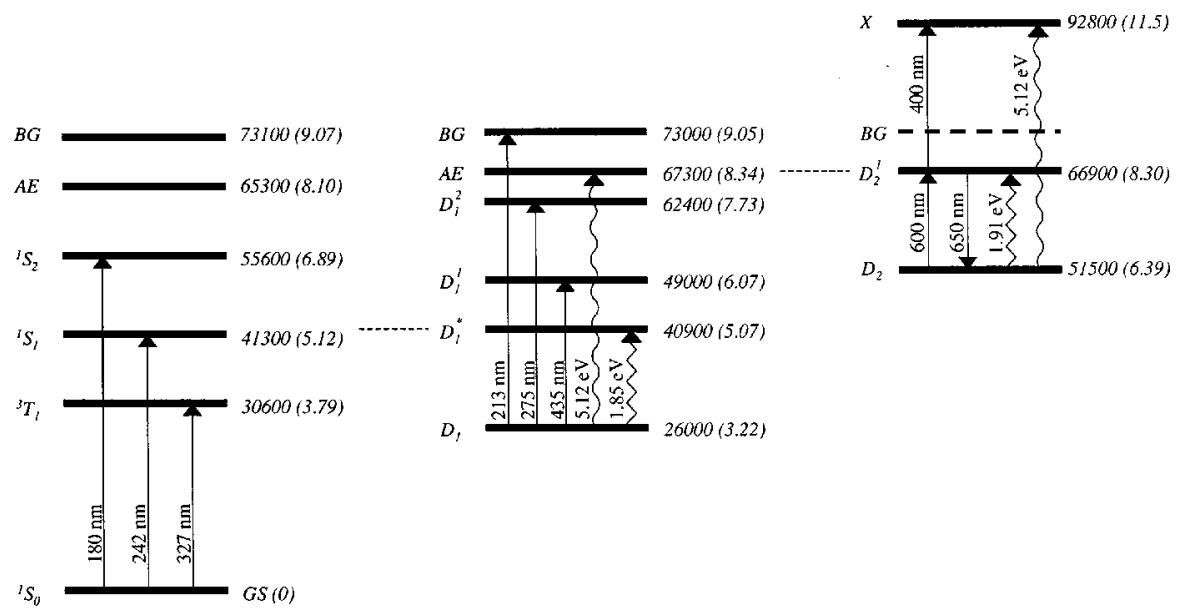

(a)

(b)

(c)

FIG. 1. Level scheme for the germanium sites (a) and the defects $D_{1}$ (b) and $D_{2}$ (c) involved in the UV-induced processes in silica glass. Energy levels are drawn as horizontal lines with the energy in $\mathrm{cm}^{-1}$ and in parentheses in electron volts (eV) to the right. Optical transitions are drawn as vertical lines labeled with the wavelength in $\mathrm{nm}$. Nonradiative transitions are shown as vertical zigzag lines with the energy in eV. Absorption edges are labeled "AE," band-gap energies labeled "BG," and the ground state labeled "GS." Dashed horizontal lines indicate transition states. $X$ is a localized state above the band gap and resonant with the singlet-triplet transition energy in the hydrogen molecule.

cial oxygen-deficient deposition. Effects of $\mathrm{H}_{2}$ loading are included in the model, but other sensitization effects are neglected. The anneal in oxygen-enriched atmosphere, which has the opposite effect of sensitization, is also not described by the model. It is possible to include results for sensitized glass by assuming that sensitization has a catalytic effect, which leads to reduced steric hindrance of all the induced processes in the glass or to increased absorption near 242 $\mathrm{nm}$. Finally, it is possible to use the model to get quantitative agreement with anneal experiments performed on gratings in different fibers and to extract activation energies and trial rates for thermal elimination of the two types of UV-induced defects.

In the following the model will be described in detail. Then five examples will be given on how to use it to analyze experimental results. During this procedure the free parameters in the model will be determined. Finally, some predictions of effects will be given.

\section{ENERGY LEVELS AND TRANSFER MECHANISMS}

The most basic assumption of the model is that energy is initially absorbed in close vicinity of the germanium atoms and later transferred to other sites in the glass where defects are created. In this respect the new model belongs to the class of microscopic models. ${ }^{3-7}$ It is well known that germanium sites in silica have an absorption spectrum consisting of two strong singlet-singlet transitions near $242 \mathrm{~nm}$ and 180 nm. ${ }^{17}$ The 242-nm absorption is strong for oxygen-deficient germanium-doped silica [e.g., with germanium-related oxygen-deficient centers (GODC's) such as two-coordinated germanium $^{18}$ ] and very weak or absent for fully oxidized silica. In addition, there is a weak singlet-triplet transition near $327 \mathrm{~nm}$. Most UV writing uses wavelengths near the
$242 \mathrm{~nm}$ absorption, ${ }^{19-21}$ some work has been performed with 193-nm excimer lasers making use of the tail of the 180-nm absorption, ${ }^{22,23}$ and a few experiments have been performed near 327 nm. $^{24}$ In Fig. 1(a) these observations are collected in a simple level scheme for germanium sites with a ground state defined at $0 \mathrm{~cm}^{-1}$ and excited states at $30600 \mathrm{~cm}^{-1}$ $\left({ }^{3} T_{1}\right), 41300 \mathrm{~cm}^{-1}\left({ }^{1} S_{1}\right)$, and $55600 \mathrm{~cm}^{-1}\left({ }^{1} S_{2}\right)$. The states are broad due to phonon broadening and the existence of different germanium sites. 5

The model assumes that two metastable defect sites with long lifetimes exist in the glass. One of these defects is responsible for positive index changes. Based on the arguments and experimental data presented below it is probably situated near $26000 \mathrm{~cm}^{-1}$. This defect will be named $D_{1}$, where $D$ stands for defect as illustrated in Fig. 1(b). The $D_{1}$ defect is possibly related to the nonbridging oxygen hole center (NBOHC defect). ${ }^{25}$ Since large fluence is needed to induce significant refractive index changes and these are relatively stable despite the moderate activation energy, there is good reason to believe that the transition from $D_{1}$ to the ground state must involve significant rearrangement of the glass matrix close to a germanium atom and possibly a spin flip. This makes the zero-temperature excited-state lifetime virtually infinite. In practice all transitions from the defect state to the ground state take place via thermal excitation to a transition state. Analysis of results from anneal experiments and accelerated aging tests indicate that this transition state $\left(D_{1}^{*}\right)$ is situated approximately $14900 \mathrm{~cm}^{-1}(1.85 \mathrm{eV})$ higher at $40900 \mathrm{~cm}^{-1}$ as will be elucidated later. This is close in energy to the excited germanium-site state ${ }^{1} S_{1}$. The close energy match means that excitation energy may easily be transferred between these states through a nonradiative transition. This is actually the main reason to assign the en- 
ergy $26000 \mathrm{~cm}^{-1}$ to the $D_{1}$ state. In addition to the $D_{1}^{*}$ transition state, which is hard to reach directly due to steric hindrance, there are other excited states of the $D_{1}$ defect. If one measures the absorption spectrum of UV-irradiated silica glass with positive index changes, one finds some complicated changes in the absorption near $242 \mathrm{~nm} \cdot{ }^{26}$ In addition there is a weak absorption near $435 \mathrm{~nm}$ and a very weak absorption near $600 \mathrm{~nm} .{ }^{27,28}$ The complicated changes in the absorption near $242 \mathrm{~nm}$ have been assigned to the combination of an absorption near $275 \mathrm{~nm}$, another absorption centered at $213 \mathrm{~nm}$, and a reduced absorption at $242 \mathrm{~nm} \cdot{ }^{26}$ Some papers make a more detailed distinction between different contributions. The increased absorption below $242 \mathrm{~nm}$ may well be due to the absorption edge reached from the defect state $D_{1}$, and $213 \mathrm{~nm}$ will then correspond to excitation to the top of the band gap. This is a considerably simpler and more straightforward interpretation than the assignments sometimes found in other papers ${ }^{26}$ and an additional reason to assign the energy $26000 \mathrm{~cm}^{-1}$ to $D_{1}$. The $435-\mathrm{nm}$ and 275-nm absorptions are most likely due to excited defect states. If this is correct, there should be states $D_{1}^{1}$ at 49000 $\mathrm{cm}^{-1}$ and $D_{1}^{2}$ at $62400 \mathrm{~cm}^{-1}$. UV-induced index changes at $193 \mathrm{~nm}$ may well proceed through interactions with these states. When using the value $26000 \mathrm{~cm}^{-1}$ for the $D_{1}$ energy and assuming complete structural relaxation after excitation above the band gap, the $D_{1}$ absorption edge is predicted to be at $67300 \mathrm{~cm}^{-1}$, and the band-gap energy is found at $73000 \mathrm{~cm}^{-1}$, which is in perfect agreement with the known band-gap energy in silica.

The 600-nm absorption is assumed to be due to a second metastable defect $D_{2}$, which may be responsible for negative index changes. ${ }^{29,30}$ Negative index changes only occur in stable, germanium-doped glass if it is exposed for a very long time or if very intense UV light close to the damage threshold irradiates it. When using very high intensity, negative index changes may be obtained with a moderate fluence. During very long exposures with moderate intensity one first observes the normal positive index change and later (after many hours or even days of exposure) a negative index change. However, various unstable or overdense types of silica may show negative index changes after short time even under normal experimental conditions. ${ }^{31}$ These observations indicate that negative index changes cannot be made directly, but must occur through some transition state, which may either be multiply excited (as is probably the case when using extreme intensity) or, for instance, the above-mentioned defect state $D_{1}$. This can be explained conveniently if the $D_{2}$ defect state is either formed by further rearrangement, if it is a high-spin state, or both. The best agreement and selfconsistency are obtained in the first case. It is of course difficult to determine its exact absolute energy, but there are some rather good reference points. First of all, data from thermal elimination of UV-induced defects ${ }^{13}$ point to a transition state around $15400 \mathrm{~cm}^{-1}(1.91 \mathrm{eV})$ above the metastable $D_{2}$ state. This is, by the way, quite close to the abovementioned 600-nm absorption and an emission observed at $650 \mathrm{~nm}$ during very long UV exposures. ${ }^{32-34} \mathrm{In}$ analogy with the situation for the first defect state it is likely that the tran- sition state is close to resonance with an energy level which may be excited near $242 \mathrm{~nm}$ from $D_{1}$, probably through a nonradiative transfer of excitation energy from a germanium site. This means around $67000 \mathrm{~cm}^{-1}$. This is close to the absorption edge and it is possible that the two are identical. I therefore estimate that the $D_{2}$ energy is $51500 \mathrm{~cm}^{-1}$ as illustrated in Fig. 1(c). This immediately fixes the first excited state $D_{2}^{1}$ to $66900 \mathrm{~cm}^{-1}$. This can be reached from $D_{1}$ through a nonradiative dipole-dipole excitation followed by a radiative or nonradiative decay. Finally, there is reason to believe that excitation energy from 242-nm light may excite $D_{2}$ defects to some state near $92800 \mathrm{~cm}^{-1}(11.5 \mathrm{eV})$, well above the band gap as will be discussed later. This energy is by the way close to resonance with the singlet-triplet transition energy in the hydrogen or deuterium molecule (11.8 $\mathrm{eV})$. This coincidence may be an important part of the catalytic effect of hydrogen loading, since it facilitates the UVinduced elimination of index reducing defects. The effect is greatly enhanced by heating which excites some of the molecules to their first vibration level $(v=1)$ and effectively reduces the $\mathrm{H}_{2}$ singlet-triplet transition energy to $11.5 \mathrm{eV}$.

\section{RATE EQUATIONS FOR UV-INDUCED TRANSITIONS}

Based on the assumptions and assignments above it is possible to write down rate equations describing the UVinduced processes. Ideally these equations should describe all observations of UV-induced changes, both dynamic and static, and the thermal decay. In addition one may expect that they will agree with the observed optical luminescence during UV writing and the induced changes in absorption. Finally, one may hope that they can predict new effects.

The concentration of the index increasing defect $D_{1}$ will be named $x$ and the concentration of the index decreasing defect $D_{2}$ will be named $y$. Assuming that $y \ll x<1$ it is possible to write simple rate equations for the defect concentrations at different positions in the glass:

$$
\begin{gathered}
\frac{d x}{d t}=c_{1}(1-x)-c_{2} x, \\
\frac{d y}{d t}=c_{3} x-c_{4} y .
\end{gathered}
$$

This gives the time dependence of the defect concentrations,

$$
\begin{aligned}
x=x_{0} \exp \left[-\left(c_{1}+c_{2}\right) t\right]+\frac{c_{1}}{c_{1}+c_{2}}\left\{1-\exp \left[-\left(c_{1}+c_{2}\right) t\right]\right\} \\
y=y_{0} \exp \left(-c_{4} t\right)+\frac{c_{1} c_{3}}{\left(c_{1}+c_{2}\right) c_{4}}\left\{1-\exp \left(-c_{4} t\right)\right\} \\
+\frac{c_{3}}{c_{4}-c_{1}-c_{2}}\left(x_{0}-\frac{c_{1}}{c_{1}+c_{2}}\right)\left\{\exp \left[-\left(c_{1}+c_{2}\right) t\right]\right. \\
\left.-\exp \left(-c_{4} t\right)\right\}
\end{aligned}
$$

where $x_{0}$ is the initial concentration of the index increasing defect $D_{1}$ and $y_{0}$ is the initial concentration of the index decreasing defect $D_{2}$. Here $c_{1}, c_{2}, c_{3}$, and $c_{4}$ are parameters 
which describe contributions from different excitation migration processes ${ }^{35,36}$ which convert photon energy initially absorbed at germanium sites to internal energy in the form of defects in the glass.

The first question is now which kind of interaction mechanism transfers the energy from the germanium site to the place where the $D_{1}$ defect is formed. The simplest choice is to assume a dipole-dipole interaction (with $r^{-6}$ dependence as a function of distance from the excited site) as is often done with success in chemical models. ${ }^{35}$ However, there are in principle many other possibilities. These include dipole-quadrupole $\left(r^{-8}\right)$, quadrupole-quadrupole $\left(r^{-10}\right)$, and direct electronic interaction $\left(e^{-k r}\right){ }^{14,15,37}$ During the development of the model, I have tried each of these possibilities to describe the radial dependence of the $c$ parameters. It turned out that only the dipole-quadrupole interaction gives a correct description of the scaling of the UV-induced refractive index changes with germanium concentration. It also allows the best agreement with other experimental observations. This is of course an important argument in favor of the dipole-quadrupole mechanism, but it is desirable if one can give independent arguments for why it could be like this. One way to get a dipole-quadrupole mechanism is if one of the interactions is spin forbidden and the defect state resembles an atomic $L S$-coupling state. ${ }^{38}$ However, most defects are somewhat delocalized electronic states of molecular nature for which it is impossible to use atomic $L S$-coupling arguments to establish a direct link between spin forbidden transitions and the $r^{-8}$ dependence $e^{14,37}$ unless the defect states have special symmetries. ${ }^{39-41}$ The transition may therefore be spin forbidden but still dipole allowed. Independent of the details, the excited-state lifetime will increase as $1 / \alpha_{F}^{2}$, where $\alpha_{F}$ is the fine structure constant. This is important to allow sufficient time for a rearrangement of the local glass structure while the electronic excitation is present. It is therefore possible that $D_{1}^{*}$ and $D_{1}$ may be triplet states and that this can be the reason for the $r^{-8}$ dependence.

Based on these arguments, I believe that the most important contribution to the formation of $D_{1}$ defects, as described by $c_{1}$, is a nonradiative dipole-quadrupole process which transfers the germanium-site excitation energy into the generation of an excited defect nearby. I therefore name the model the "UV dipole-quadrupole model." The dipolequadrupole process is catalyzed by hydrogen for two reasons. First of all, hydrogen increases the absorption near 242 $\mathrm{nm}$, particularly after thermal activation. Second, hydrogen is generally known to catalyze many processes in the glass, particularly those which involve rearrangement ${ }^{42}$ such as the transition from $D_{1}^{*}$ to $D_{1}$.

In addition to the dipole-quadrupole effect there will also be a nonlinear contribution from two-photon processes creating highly excited states well above the band gap using a singly excited germanium site as resonant intermediate state. Because the density of states is very high above the band gap, the whole process will probably be of resonant nature. This will lead to a long-range behavior ${ }^{43}$ possibly in the form of a resonant dipole-quadrupole process. It could also involve long-range effects from free charges, but this will not be considered further here.
Annihilation of $D_{1}$ defects, described by the parameter $c_{2}$, can take place in two ways. The first possibility is through a second dipole-quadrupole process which creates a state near the band-gap energy. This will quickly decay to the singlet ground state. A second possibility is that a $D_{2}$ defect is generated. This particular process is described by $c_{3}$. It will most likely take place through a dipole-dipole interaction with an excited germanium site. However, at very high laser power it may also happen through a three-photon mechanism initiating a similar process via the continuum.

Annihilation of $D_{2}$ defects can take place in three different ways. The first possibility is another dipole-quadrupole process with an excited germanium site. This creates a shortlived state well above the band gap. A second possibility is that a relatively long-lived state $X$ exists in this area. $X$ may then be reached either by position-independent direct optical excitation or by a resonant dipole-dipole process which is also of long-range nature. ${ }^{43}$ In conclusion, $c_{1}, c_{2}, c_{3}$, and $c_{4}$ are given by

$$
\begin{gathered}
c_{1}=\frac{\epsilon}{r^{8}}\left[A s_{1}\left(1+\kappa_{1} P_{H_{2}}\right)+\alpha_{2} r^{4} I_{\text {laser }}^{2}\right] \\
c_{2}=\frac{\epsilon}{r^{8}}\left\{A\left[s_{2}\left(1+\kappa_{2} P_{H_{2}}\right)+s_{3}\left(1+\kappa_{3} P_{H_{2}}\right) r^{2}\right]+\alpha_{3} r^{2} I_{\text {laser }}^{3}\right\} \\
+k_{x}\left(\frac{r_{1}}{r}\right)^{10} \exp \left(-\frac{\Delta E_{x}}{R T}\right), \\
c_{3}=\frac{\epsilon}{r^{8}}\left\{A\left[s_{2} \kappa_{2} P_{H_{2}}+s_{3}\left(1+\kappa_{3} P_{H_{2}}\right) r^{2}\right]+\alpha_{3} r^{2} I_{\text {lase }}^{3}\right\} \\
c_{4}=\epsilon A\left[\frac{s_{4}}{r^{8}}+\left(\frac{s_{5}}{r^{3}}+s_{6}\right)\left(1+\kappa_{4} P_{H_{2}}\right)\right] \\
+k_{y}\left(\frac{r_{1}}{r}\right)^{10} \exp \left(-\frac{\Delta E_{y}}{R T}\right),
\end{gathered}
$$

where $r$ is the distance from the nearest germanium atom, $\epsilon=\Gamma \alpha_{F}^{2}=\Gamma / 137^{2}$ is the probability for electric quadrupole or spin-forbidden transitions, $\Gamma$ is the decay rate for an allowed optical transition, $A$ is the optical excitation probability for the initial transition, $s_{1}, s_{2}, s_{3}, s_{4}, s_{5}$, and $s_{6}$ are the branching rates (including the effect of steric hindrance during rearrangement), $\kappa_{0}, \kappa_{1}, \kappa_{2}, \kappa_{3}, \kappa_{4}$, and $\kappa_{5}$ are the hydrogen catalysis coefficients, $P_{\mathrm{H}_{2}}$ is the $\mathrm{H}_{2}$ loading pressure with which equilibrium has been obtained at room temperature, $\alpha_{2}$ is the two-photon probability, $\alpha_{3}$ is the threephoton probability, $k_{x}$ and $k_{y}$ are the thermal trial rates, $r_{1}$ is the distance to the nearest neighbor, $\Delta E_{x}$ and $\Delta E_{y}$ are the thermal activation energies, $R$ is the gas constant, and $T$ is the absolute temperature. It is assumed that the two-photon transition from the ground state is resonant $\left(\propto r^{-4}\right)$ but weak, since it can reach above the band gap of silica where mixing is likely and the level density is high but the overlap integrals are small. It is also assumed that excitations of $D_{1}$ to the $D_{2}$ system takes place through simple dipole-dipole 
transitions $\left(\propto r^{-6}\right)$ followed by a decay at the local site. Another possibility is to annihilate $D_{1}$ defects directly by dipole-quadrupole transfer of energy from an excited germanium site. This is described by the coefficient $s_{2}$. Hydrogen loading may catalyze some of these excitations to decay in a different way, leading to the formation of $D_{2}$ defects. This is described by $\kappa_{2}$. Excitation of the $D_{2}$ defect at $242 \mathrm{~nm}(5.12$ $\mathrm{eV}$ ) brings it well above the band gap which leads to its elimination. Therefore, the elimination mechanism is either a direct optical transition to a relatively long-lived excited state $X$ as described by $s_{6}$ or a resonant dipole-dipole transition $\left(\propto_{5} r^{-3}\right)$ to the same state with the energy absorbed by a germanium site nearby. Because the level $X$ and the singlettriplet transition in the hydrogen molecule are almost resonant, the decay of $X$ is strongly enhanced by the presence of hydrogen as described by $\kappa_{4}$. In analogy with the situation for the $D_{1}$ defect there is also the possibility of annihilation through a nonresonant dipole-quadrupole transition which is described by $s_{4}$. Finally, it is assumed that all thermal eliminations take place through quadrupole-quadrupole interactions $\left(\propto r^{-10}\right)$ with transitions in the central germanium atom. This last choice is somewhat arbitrary since the reaction path is not known in detail. However, satisfactory agreement with experimental data can only be achieved with exponents greater than or equal to 10 . The optical excitation probability $A$ near $242 \mathrm{~nm}$ for an oxygen-deficient germanium site is given by

$$
A=\frac{I_{\text {laser }} \exp \left[-\left(\frac{\lambda-\lambda_{a b s}}{\Delta \lambda_{a b s}}\right)^{2}\right]\left(1+\kappa_{0} P_{H_{2}}\right)}{I_{\text {sat }}+2 I_{\text {laser }} \exp \left[-\left(\frac{\lambda-\lambda_{a b s}}{\Delta \lambda_{a b s}}\right)^{2}\right]\left(1+\kappa_{0} P_{H_{2}}\right)} P_{\text {laser }},
$$

where $I_{\text {laser }}$ is the UV laser intensity, $\lambda$ is the laser wavelength, $\lambda_{a b s}$ is the center wavelength for the germanium site absorption, $\Delta \lambda_{a b s}$ is the spectral width of the absorption (having almost Gaussian shape), $I_{\text {sat }}$ is the effective saturation intensity for the absorption, and $P_{\text {laser }}$ is the fraction of the writing time with the laser on (only relevant when using pulsed lasers). Hydrogen loading is presumed to catalyze the absorption both because of the presence of a nearby $\mathrm{OH}$ transition $^{44}$ and because it may modify the germanium sites in such a way that they become oxygen deficient and increase the 242-nm transition probability. Both these catalytic effects may demand initial activation and therefore be facilitated by heat treatment. The multiphoton probabilities are given by

$$
\alpha_{2}=\alpha_{2,0}\left(1+\kappa_{5} P_{H_{2}}\right)^{2} P_{\text {laser }}, \quad \alpha_{3}=\alpha_{3,0} P_{\text {laser }},
$$

where $\alpha_{2,0}$ and $\alpha_{3,0}$ are the unperturbed probabilities without hydrogen loading. Hydrogen catalysis of the three-photon process is ignored since no plausible mechanism exists for it and since the available experimental data indicate no such effect. Because the probabilities for energy transfer decay so rapidly with distance $r$, it is normally not necessary to take into account the influence of second nearest neighbors. However, two exceptions are the resonant dipole-dipole annihila- tion of $D_{2}$ states $\left(\propto r^{-3}\right)$ mediated by the $\mathrm{H}_{2}$ triplet state and the resonant two-photon dipole-quadrupole excitation from the ground state $\left(\propto r^{-4}\right)$. In these cases it is necessary to replace $r^{-3}$ and $r^{-4}$ with

$$
\begin{aligned}
& \frac{1}{r^{3}}\left[1+\left(\frac{r}{2 r_{\text {max }}-r}\right)^{3}\right], \\
& \frac{1}{r^{4}}\left[1+\left(\frac{r}{2 r_{\text {max }}-r}\right)^{4}\right],
\end{aligned}
$$

respectively to include first-order effects of the next-nearest neighbor. $r_{\max }$ is given by

$$
\begin{gathered}
V_{G e}-V_{m}=4 \pi \int_{0}^{r_{\max }} \rho(r) r^{2} d r, \\
V_{m}=\frac{M_{\mathrm{SiO}_{2}}}{3 \rho_{\mathrm{SiO}_{2}} N_{A}}, \\
V_{G e}=\frac{100 M_{\mathrm{SiO}_{2}}}{n_{G e} \rho_{\mathrm{SiO}_{2}} N_{A}}, \\
n_{G e}=100 \frac{N_{G e}}{N_{\mathrm{Si}}+N_{\mathrm{Ge}}},
\end{gathered}
$$

where $\rho(r)$ is the radial density function starting in an average germanium atom, $V_{G e}=V_{\text {sample }} / N_{G e}$ is the germanium molar volume (assuming the germanium atoms share the entire volume of the sample, $\left.V_{\text {sample }}\right), V_{m}$ is the average molar volume (including all atoms), $\mathrm{MSiO}_{2}$ is the molar mass of $\mathrm{SiO}_{2}, \quad \rho_{\mathrm{SiO}_{2}}$ is the density of undoped silica, $N_{A}$ is Avogadro's number, $n_{G e}$ is the mole \% of germanium, $N_{G e}$ is the number of germanium atom's and $N_{S i}$ is the number of silicon atoms.

It is now possible to calculate the index change by integration over the germanium molar volume. During this integration it is of course implicitly assumed that the distribution of germanium atoms is equidistant. This may not be entirely correct, but it is the simplest and most reasonable approximation. The result can formally be written

$$
\Delta n=\frac{4 \pi}{V_{G e}-V_{m}} \int_{0}^{r_{\max }}\left[\Delta n_{\max }^{x} x(r)+\Delta n_{\max }^{y} y(r)\right] \rho(r) r^{2} d r,
$$

where $\Delta n_{\max }^{x}$ is the index change induced by unit density of index increasing defects and $\Delta n_{\max }^{y}$ is the index change induced by unit density of index decreasing defects. The radial density function $\rho(r)$ is determined from experimental x-ray diffraction data for silica ${ }^{45,46}$ starting at a silicon atom, e.g., the sum of the $\mathrm{Si}-\mathrm{Si}$ and $\mathrm{Si}-\mathrm{O}$ pair function distribution curves. This implies that the defect generation is considered to be equally likely at all atoms in the glass. There is no basic reason to make this assumption, but trial and error has shown that it is the only way to obtain good agreement with the experimental data. This becomes particularly obvious when 
considering the red luminescence curves described below, where any other assumption would not agree with the number of steps and their observed height. ${ }^{32,47}$ It may be an important clue to the exact nature of the defect states.

When the local index change is determined using Eq. (8) it is possible to calculate the effective index change in fibers or waveguides and the strength of Bragg gratings. One particularly interesting case is the strength $S$ of a uniform Bragg grating of length $L_{\text {exp }}$. Conventionally this is measured as the relative reduction (in $\mathrm{dB}$ ) of the transmitted light on resonance which is given by

$$
S=-10 \log _{10}\left[1-\tanh ^{2}\left(\frac{\pi \eta\left|\Delta n_{\text {max }}-\Delta n_{\text {min }}\right|}{2\left(n_{\text {eff }}+\eta \Delta n_{\text {avg }}\right) \Lambda_{\text {mask }}} L_{\text {exp }}\right)\right],
$$

where $\Delta n_{\min }$ and $\Delta n_{\max }$ are the index changes at minimum and maximum local intensity in the UV interference pattern with visibility $v_{g}$ formed by a phase mask with period $\Lambda_{\text {mask }}, \Delta n_{\text {avg }}$ is the average index change, $\eta$ is the confinement factor (core overlap) for the waveguide mode, and $n_{\text {eff }}$ is the effective refractive index for the waveguide mode.

Another important aspect of the UV induced processes is the luminescence emitted during UV exposure. It is an important test of the quality of any model for UV-induced effects that it is able to describe the properties of the luminescence. During most exposures of germanium-doped silica some kind of visible luminescence is emitted. An exception is silica annealed in oxygen-enriched atmosphere for prolonged time. In this case the 242-nm absorption is very weak and virtually no luminescence is emitted. At the same time the refractive index remains practically unchanged. Ignoring this special case for a moment, the predominant luminescence from germanium-doped silica is blue with a relatively narrow spectrum around $400 \mathrm{~nm}$. It is believed to be due to optical decay at GODC sites ${ }^{48,49}$ probably through the triplet state $\left({ }^{3} T_{1} \rightarrow{ }^{1} S_{0}\right.$ at the emission site). The blue luminescence decays gradually after some exposure time following a curve similar to a stretched exponential function plus a constant. ${ }^{32,50}$ When the silica is loaded with a high concentration of hydrogen, a spectrally broad (white) luminescence is sometimes observed. ${ }^{27}$ It does not decay with exposure time before the hydrogen diffuses out and it almost looks like a blackbody Planck spectrum corresponding to a temperature of more than $2000 \mathrm{~K}$. Based on the model in this paper it is very likely that this spectrum is the luminescence from the metastable triplet state in the hydrogen molecule generated during destruction of $D_{2}$ defects to the repulsive $b$ state. Finally, after very long exposures an extremely weak red luminescence occurs. It has a relatively narrow spectrum around $650 \mathrm{~nm}$. I believe it is due to optical decay of excited $D_{2}$ defects. In addition to these types of visible luminescence some groups have observed UV luminescence. ${ }^{49}$ The UV luminescence is most likely due to in-band luminescence from the 242-nm band.

Here I will only consider the blue and red luminescence in detail. There are five factors determining the intensity of these two types of luminescence. These are the probability of exciting the upper state, the nonradiative quenching due to the presence of other defects, the number of available sites, the collection efficiency, and the absorption loss. Starting with the last two and simplest parts, the combined effect of collection efficiency and absorption loss is determined by the numerical aperture, the length of the exposed area, $L_{\text {exp }}$, and the absorption per unit length, $\alpha_{\lambda}$. Most observations have been made in fibers and waveguides with numerical apertures in a narrow interval, so no further treatment will be given of this factor. The combined effect of evenly distributed emission and absorption loss during uniform exposure of $L_{\text {exp }}$ gives a prefactor

$$
\frac{1}{\alpha_{\lambda}}\left[1-\exp \left(-\alpha_{\lambda} L_{\exp }\right)\right],
$$

where $\alpha_{\lambda}$ is primarily due to the tail of the 435-nm absorption at $400 \mathrm{~nm}$ and the tail of the $600-\mathrm{nm}$ absorption at 650 $\mathrm{nm}$. This can be summarized as

$$
\begin{aligned}
& \alpha_{400}=\alpha_{400}^{0} \frac{4 \pi}{V_{G e}-V_{m}} \int_{0}^{r_{\max }} x(r) \rho(r) r^{2} d r \\
& \alpha_{650}=\alpha_{650}^{0} \frac{4 \pi}{V_{G e}-V_{m}} \int_{0}^{r_{\max }} y(r) \rho(r) r^{2} d r
\end{aligned}
$$

where $\alpha_{400}^{0}$ is the 400 -nm absorption coefficient per concentration unit for $D_{1}$ and $\alpha_{650}^{0}$ is the 650-nm absorption coefficient per concentration unit for $D_{2}$. This expression must be multiplied by the coupling coefficient $\alpha_{c p l}$ to the detection system, e.g., a standard fiber, which in the Gaussian approximation $^{51}$ is given by

$$
\alpha_{c p l}=\frac{r_{c} r_{d e t}}{2\left(r_{c}^{2}+r_{d e t}^{2}\right)^{2}},
$$

where $r_{c}$ is the core radius and $r_{d e t}$ is the core radius for the interfacing detection fiber $(4.4 \mu \mathrm{m}$ for standard SMF28 fiber). For silica which is not annealed in oxygen it is a good assumption that the number of available sites for induced luminescence will be proportional to the germanium atom density per unit length, $\pi r_{c}^{2} n_{G e}$. For the blue luminescence the quenching is due to nonradiative dipole-dipole interaction with $D_{1}$ defects and therefore the emission rate is proportional to

$$
P_{\text {blue }}^{q}=\frac{1}{1+\alpha_{\text {blue }}^{q}\left(x-x_{n n}\right) r^{-6}},
$$

where $\alpha_{b l u e}^{q}$ is the quenching factor and $x_{n n}$ is the number of nearest neighbor $D_{1}$ defects. The nearest neighbors are coupled so strongly to the site that their effect is different. The best agreement with experiments is obtained if it is assumed that radiative decay is still the most probable decay mechanism for these nearest neighbors. For all other defects nonradiative decay will dominate. The red luminescence is due to optical decay of $D_{2}$ defects from their first excited level to their ground level. Excitation of $D_{2}$ defects to their second excited level takes place via direct optical excitation or via a resonant dipole-dipole interaction with an optically 
excited germanium site. Population of the first excited level takes place through radiative or nonradiative decay which is catalyzed by nearby $D_{1}$ defects through their first transition which is almost resonant in energy with the transition between the first and second excited levels of the $D_{2}$ defects. In addition there will be a quenching effect due to nearby $D_{2}$ defects which tend to mix the first excited states near the absorption edge in such a way that nonradiative decay is facilitated. Another effect with similar consequence is the catalysis of nonradiative transitions to the ground level of $D_{2}$ via Auger mechanisms involving levels above the band gap if both $D_{2}$ defects are excited simultaneously. ${ }^{52}$ This can be summarized as

$$
I_{r e d} \propto \int_{0}^{r_{\max }} y(r)\left(\frac{1}{r^{3}}+\frac{s_{6}}{s_{5}}\right) \frac{1+r_{c a t} x_{n n}}{1+\alpha_{r e d}^{q}\left(y-y_{n n}\right)} \rho(r) r^{2} d r,
$$

where $r_{\text {cat }}$ is the factor for $D_{1}$ catalysis of the transition from second to first excited level in $D_{2}, \alpha_{r e d}^{q}$ is the $D_{2}$ quenching factor for the red transition, and $y_{n n}$ is the local population of $D_{2}$ defects (which must be subtracted since there is no selfquenching). For the nearest neighbors to the germanium site the catalysis is dominated by catalytic effects from the site. These effects must be closely related to the blue luminescence. For simplicity I take this into account by replacing $r_{c a t} x_{n n}$ by $P_{b l u e}^{q}$ for the nearest neighbors. Finally, the excitation probability for both the blue and red luminescence is proportional to the optical excitation probability for germanium sites, $A$, and the total luminescence is given by proportionality factors $A_{\text {blue }}$ for the blue luminescence and $A_{\text {red }}$ for the red luminescence.

\section{RESULTS}

Equation (2) describes the local defect density induced by UV light in germanium-doped silica. The average properties of the glass can be calculated by integrating this over the entire volume using the interpretations in Eq. (3). Equations (4)-(14) can then be used to calculate various properties of the glass and of particular fibers or waveguides irradiated by UV light. Integration over the radial coordinate is performed using Romberg's method of order $2 K$, where $K=2$ is Simpson's rule, ${ }^{53}$ to an accuracy around $10^{-10}$. During UV exposures at room temperature the thermal contributions to Eqs. (3) can often be ignored. On the other hand, all contributions except the thermal ones can normally be ignored during decay and accelerated testing. It is therefore relatively simple to use Eqs. (2) and (3) to make an additional integration over the thermal history of a sample and predict decay curves.

In total the model has 25 free parameters and 7 fixed parameters which can be estimated from first principles. Approximate values of the free parameters have been determined in such a way that a few selected UV experiments are reproduced well. This includes five different UV exposures following the change in effective refractive index, the strength of UV-induced gratings, and the luminescence. Two of these exposures were performed using unloaded fiber, low
UV power, and widely different germanium concentrations in the core (3.2\% and 25\%). One measurement of the red and blue luminescence in standard fiber (3.2\% germanium) with intermediate UV power $\left(6 \mathrm{~kW} / \mathrm{cm}^{2}\right)$ and short exposure length $L_{\text {exp }}$ was used to determine the parameters for the visible luminescence without significant influence from photodarkening during the exposure. ${ }^{32,54,55}$ The fourth measurement used for optimization of the exposure parameters was performed at high power and high germanium concentration $(22.8 \%)$, primarily assessing the nonlinear effects. In addition, one experiment with hydrogen-loaded standard fiber was used to adjust the $\kappa$ parameters related to the influence of hydrogen loading. $\kappa_{5}$ was found by adjusting it to fit the observed refractive index change during an excimer laser exposure of deuterium loaded fiber with $9 \%$ germanium in the core. Finally, the optimization included the result of one accelerated aging experiment ${ }^{13}$ for a fiber with intermediate germanium concentration in the core $(15 \%)$ in order to determine the thermal parameters. The resulting parameter values are collected in Table I. It should be noted that no special curve fitting was used to optimize the parameter values, so further improvement may well be possible using, e.g., leastsquares fitting to a large number of experimental results. The key parameters for the selected spectra used for the optimization are listed in Table II.

The first basic test of the model is if it is able to reproduce the selected spectra with good accuracy and with physically acceptable values of the basic parameters. Figure 2 shows a typical example of one of these spectra. The deviations are generally $10 \%-15 \%$, sometimes less. This level of agreement is fully satisfactory considering the crude optimization of parameter values and that the experimental accuracy is around 5\%. One particularly encouraging result is that the model reproduces the very complicated and hitherto unexplained temporal dependence of the red luminescence ${ }^{32}$ as shown in Fig. 3. The model's explanation of the steps in the curve is that each step represents the contribution from one layer of neighbors to a germanium atom. The distance to these layers is determined from x-ray spectroscopy, ${ }^{46}$ so the good agreement concerning the relative time for these steps is not due to the combined effect of the many free parameters but is an intrinsic property of the model. The number of steps is given by the $\mathrm{x}$-ray pair function distribution curves and the relative timing and step height are predominantly determined by the $r^{-8}$ dependence. However, three of the free parameters can influence the exact shape of the steps and the scaling of the time axis. Here may be room for some further improvement. Another encouraging result is that the decay of the blue luminescence is well represented. It has been believed for several years that this decay follows a stretched exponential curve perhaps with an additional constant contribution. ${ }^{32,50}$ This curve form indeed gives a good fit to most spectra. The present model predicts instead that the curve form is a radial integral over an exponentially saturating quenching contribution. This curve fits qualitatively even better to the data, since some very small kinks experimentally observed in the luminescence curve are reproduced due to the radial variation of the density. In addition a parameter set of only three parameters for the blue luminescence is able 
TABLE I. List of the parameters for the model determined by optimizing its performance for a few selected UV exposures listed in Table II and one accelerated aging experiment described in Ref. 13. The fixed parameters were estimated from first principles.

Fixed parameters

$\begin{array}{lc}\lambda_{a b s} & 242 \mathrm{~nm} \\ \Delta \lambda_{a b s} & 15 \mathrm{~nm} \\ I_{\text {sat }} & 10^{11} \mathrm{~W} / \mathrm{m}^{2} \\ \Gamma & 10^{9} \mathrm{~s}^{-1} \\ \Delta n_{\max }^{x} & 0.04 \\ \Delta n_{\max }^{y} & -0.1 \\ r_{1} & 1.61 \AA\end{array}$

Branching ratios

$\begin{array}{ll}s_{1} & 0.65 \\ s_{2} & 8.35 \\ s_{3} & 0.0145 \\ s_{4} & 0.2 \\ s_{5} & 0.0035 \\ s_{6} & 0.00002\end{array}$

Loading parameters

Non-linear parameters

$\begin{array}{ll}\kappa_{0} & 0.0085 \mathrm{~atm}^{-1} \\ \kappa_{1} & 0.3 \mathrm{~atm}^{-1} \\ \kappa_{2} & 0.06 \mathrm{~atm}^{-1} \\ \kappa_{3} & 0.06 \mathrm{~atm}^{-1} \\ \kappa_{4} & 30 \mathrm{~atm}^{-1} \\ \kappa_{5} & 0.0065 \mathrm{~atm}^{-1}\end{array}$

\begin{tabular}{lll} 
& $\alpha_{2,0}$ & $1.25 \times 10^{-24} \mathrm{~m}^{4} / \mathrm{W}^{2}$ \\
& $\alpha_{3,0}$ & $5.1 \times 10^{-36} \mathrm{~m}^{6} / \mathrm{W}^{3}$ \\
Luminescence parameters & & \\
& $A_{\text {blue }}$ & $1.35 \times 10^{2} \mathrm{~W} / \mathrm{m}^{3} / \% \mathrm{Ge}$ \\
& $A_{\text {red }}$ & $4.1 \times 10^{3} \mathrm{~W} / \mathrm{m}^{3} / \% \mathrm{Ge}$ \\
& $\alpha_{\text {blue }}^{q}$ & 675.0 \\
& $\alpha_{\text {red }}^{q}$ & 1.7 \\
& $r_{\text {cat }}$ & 2.25 \\
& $\alpha_{400}^{0}$ & $1.0 \times 10^{3} \mathrm{~m}^{-1}$ \\
& $\alpha_{650}^{0}$ & $0.5 \times 10^{3} \mathrm{~m}^{-1}$ \\
& & \\
Thermal parameters & $\Delta E_{x}$ & $(1.85 \pm 0.15) \mathrm{eV}$ \\
& $\Delta E_{y}$ & $(1.91 \pm 0.15) \mathrm{eV}$ \\
& $k_{x}$ & $(2.7 \pm 1.9) \times 10^{13} \mathrm{~Hz}$ \\
& $k_{y}$ & $(7.2 \pm 4.5) \times 10^{13} \mathrm{~Hz}$ \\
\hline \hline
\end{tabular}

to reproduce most known spectra with acceptable accuracy. At the moment one typically uses four free parameters per spectrum. It should also be noted that the stretched exponential function is actually the first approximation to such a process with no typical length scale in the limit of a smooth radial distribution. ${ }^{56}$

The optimized parameter values in Table I seem to be physically reasonable. The maximum positive index change $\Delta n_{\text {max }}^{x}$ is estimated to be $4 \times 10^{-2}$ based on the assumption that the main source for the index change is the closer proximity of the UV-absorption lines because the $D_{1}$ level is situated at elevated energy in the band gap. The number is simply calculated by shifting the refractive index curve for silica by $3.22 \mathrm{eV}$ (corresponding to the $D_{1}$ excitation energy) and subtracting the original value at $1550 \mathrm{~nm}$. This estimate may well be up to $50 \%$ off because it ignores changes in absorption strength and part of the densification effects, but it is very unlikely to be as much as an order of magnitude off. Because of the correlation between the parameters, it is impossible to determine $\Delta n_{\max }^{x}$ directly from experimental data. An independent determination can only be performed if it is possible to measure the absolute concentration of defects, but for the moment the only option is to use the simple estimate from first principles. The situation is similar but somewhat worse for $\Delta n_{\text {max }}^{y}$ because there is more uncertainty about the exact nature of the $D_{2}$ defect. The simplest assumption is that it is so decoupled from most other states that one can ignore its absorption. This would yield a value of -0.45 for $\Delta n_{\text {max }}^{y}$. Some experimental data obtained under extreme conditions (long exposures with $193 \mathrm{~nm}$ ) (Ref. 57) indicate a large negative value for $\Delta n_{\text {max }}^{y}$, but not that extreme. I chose to use a crude round number of -0.1 . X-ray data fix the value of $r_{1}$ to $1.61 \AA{ }^{45,46}$ Finally, I estimate the saturation intensity $I_{\text {sat }}$ to be around $10 \mathrm{MW} / \mathrm{cm}^{2}$ and the decay rate $\Gamma$ to be around $10^{9} \mathrm{~s}^{-1}$. With these basic values fixed it is possible to obtain the values of all the free parameters. For the branching rates $s_{n}$, one naively expects values between $10^{-3}$ and 1 depending on steric hindrance. All values except $s_{2}$ and $s_{6}$ lie within this interval. There may be two possible reasons that $s_{2}$ is as large as 8.35 . One possibility is that the estimate of the front factor $\epsilon$ is too crude, since it ignores a number of geometrical factors and uses a crude value of $10^{9} \mathrm{~s}^{-1}$ for the decay rate for allowed optical transitions. There may well be more than an order of magnitude deviation from the expected range due to these reasons. Another possibility is that the estimate of $\Delta n_{\max }^{x}$ is somewhat too large. However, independent of these explanations the high value clearly indicates that most UV-excited $D_{1}$ defects decay to other states. The smallness of $s_{6}$ is of no concern since it describes a direct optical excitation with a presumed very small overlap integral. For the $\kappa$ parameters it is hard to predict exact values. However, it is expected that hydrogen loading at a few hundred atmospheres will increase most rates significantly and that catalysis of the initial process and the resonant elimination of $D_{2}$ defects are the most efficient processes. This is exactly the pattern followed by the actual values. The thermal rates and activation energies are expected to be comparable to the average of values determined for presumed broad energy distribution. ${ }^{12}$ This is indeed the case. It is expected that the higher-order coefficients $\left(\epsilon \alpha_{2,0}\right.$ and $\left.\epsilon \alpha_{3,0}\right)$ are very small but still somewhat larger than $\chi^{(\overrightarrow{3})}$ for pure silica, which is confirmed by the actual values. Finally, the coefficients for the two transitions at $400 \mathrm{~nm}$ and $650 \mathrm{~nm}$ should be of similar order of magnitude. This is also the case.

A more serious test of the model is if it is able to reproduce other spectra not used during the parameter optimization. Figure 4 shows a typical result. The agreement is generally worse than for the selected spectra. Typical deviations are $15 \%-20 \%$ and in a few extreme cases up to $50 \%$, particularly for the absolute grating strength in the beginning of 
TABLE II. Key experimental parameters for the five spectra used for optimization of the model. $n_{G e}$ is the germanium concentration in the core, $\lambda$ the laser wavelength, $P_{d u r}$ the duration of laser pulses (when adequate), and $v_{g}$ the estimated visibility of the UV pattern.

\begin{tabular}{|c|c|c|c|c|c|c|c|c|c|}
\hline Expt. & $n_{G e}(\%)$ & $\lambda(\mathrm{nm})$ & & $I_{\text {laser }}$ & $P_{d u r}$ & Rate $(\mathrm{Hz})$ & $L_{\text {exp }}(\mathrm{mm})$ & $v_{g}$ & Part used \\
\hline 1 & 3.2 & 244 & 150 & $\mathrm{~W} / \mathrm{cm}^{2}$ & $\mathrm{cw}$ & $\mathrm{cw}$ & 3.0 & 1.0 & $\begin{array}{l}\text { Grating strength } \\
\text { Index change }\end{array}$ \\
\hline 2 & 25 & 244 & 100 & $\mathrm{~W} / \mathrm{cm}^{2}$ & $\mathrm{cw}$ & $\mathrm{cw}$ & 3.0 & 1.0 & Grating strength \\
\hline 3 & 3.2 & 244 & 6 & $\mathrm{~kW} / \mathrm{cm}^{2}$ & $\mathrm{cw}$ & $\mathrm{cw}$ & 0.04 & 0.0 & Luminescence \\
\hline 4 & 22.8 & 248 & 300 & $\mathrm{~mJ} / \mathrm{cm}^{2}$ & $25 n s$ & 20 & 2.7 & 0.91 & $\begin{array}{l}\text { Grating strength } \\
\text { Index change }\end{array}$ \\
\hline 5 & 3.2 & 244 & 120 & $\mathrm{~W} / \mathrm{cm}^{2}$ & $\mathrm{cw}$ & $\mathrm{cw}$ & 4.0 & 1.0 & $\begin{array}{l}\text { Grating strength } \\
\left(\mathrm{H}_{2} \text { loading }\right)\end{array}$ \\
\hline
\end{tabular}

the exposure. This may be due to the incomplete treatment in the model of the bleaching of the 242-nm transition. A more complete treatment of the bleaching could also further improve the agreement for the red luminescence curve. The imperfect agreement with the grating strength observed in some experiments may also be due to experimental problems such as undesired chirp or non-uniformity in the grating pattern. I consider the agreement acceptable given the remarks about parameter optimization and experimental uncertainty above.

Perhaps the most interesting question is if the model is able to make predictions about new effects and future results. It is already clear that it is possible to run the parameters of any germanium-doped fiber and predict most results for standard UV-writing experiments with reasonable accuracy. The same will be possible for germanium-doped silica waveguides. These predictions make up the core applications for the model. However, the model is still limited to standard silica glass materials using germanium doping and loading

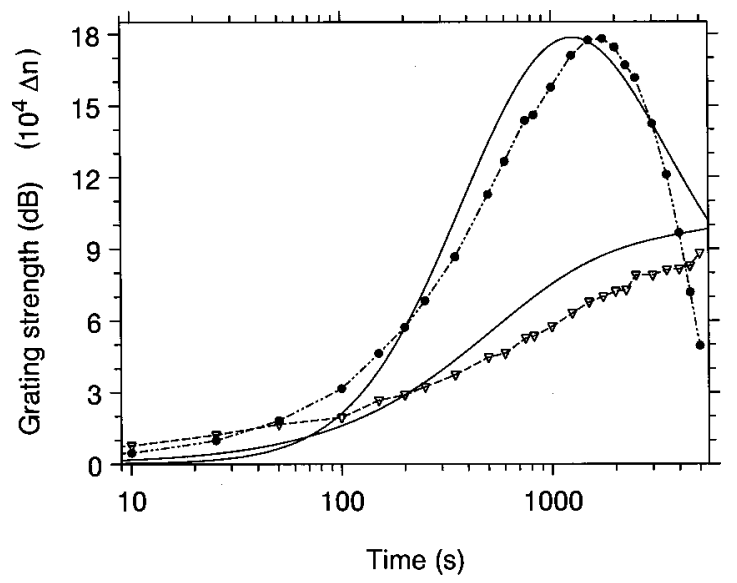

FIG. 2. One of the five spectra used for optimization of the model. The experiment was performed with an excimer laser at 248 $\mathrm{nm}, 300 \mathrm{~mJ} / \mathrm{cm}^{2}$ energy per pulse, $25-\mathrm{ns}$ pulse duration, and $20-\mathrm{Hz}$ repetition rate in an unloaded fiber with $22.8 \%$ germanium in the core. The solid circles connected with a dash-dotted line are the measured grating strengths in transmission $(\mathrm{dB})$. The triangles connected with a dashed line are the measured shifts in effective refractive index during exposure (multiplied by $10^{4}$ ). The solid lines are the predictions from the model. with hydrogen or deuterium. It does not include the effects of other dopants such as, e.g., phosphorous, aluminum, boron, or rare-earth elements, which are known to have significant effects on UV-induced index changes. It also excludes specially treated material such as glass deposited under oxygendeficient conditions, oxygen-annealed glass (unless the oxygen treatment is followed by high-pressure hydrogen or deuterium loading and activation of the gas), and glass exposed to flame brushing. 58

In this context it is probably most interesting if qualitatively new effects can be predicted. One example is the spectrally broad luminescence observed during exposure of hydrogen- or deuterium-loaded germanium-doped glass. In this case the model predicts that the spectrum should be that of the bound-free transition in the hydrogen molecule rather than a Planck spectrum. However, it should be noted that it is necessary to include some molecular corrections because the transition can only be observed in the glass host. In free

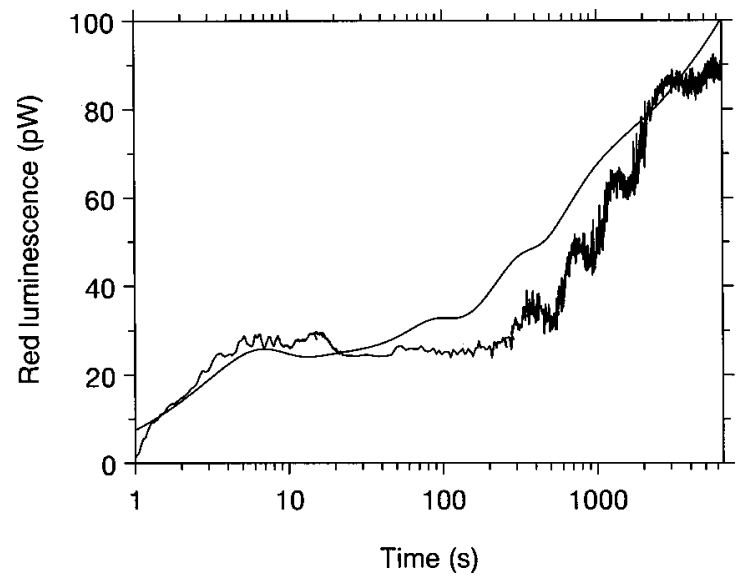

FIG. 3. The red luminescence measured with an optical spectrum analyzer for a standard fiber with $3.2 \%$ germanium in the core during an exposure with the focused spot from a 244-nm frequencydoubled argon ion (FRED) laser (Ref. 32). The intensity is $6 \mathrm{~kW} / \mathrm{cm}^{2}$ and the exposure length is $40 \mu \mathrm{m}$. The dashed, noisy line is the measured result and the full line is the prediction from the model. The relative time and height of the steps in the luminescence are reproduced well by the model even though the shape is rounded too much. The absolute timing is $\sim 50 \%$ off, perhaps due to difficulties measuring the laser spot size. 


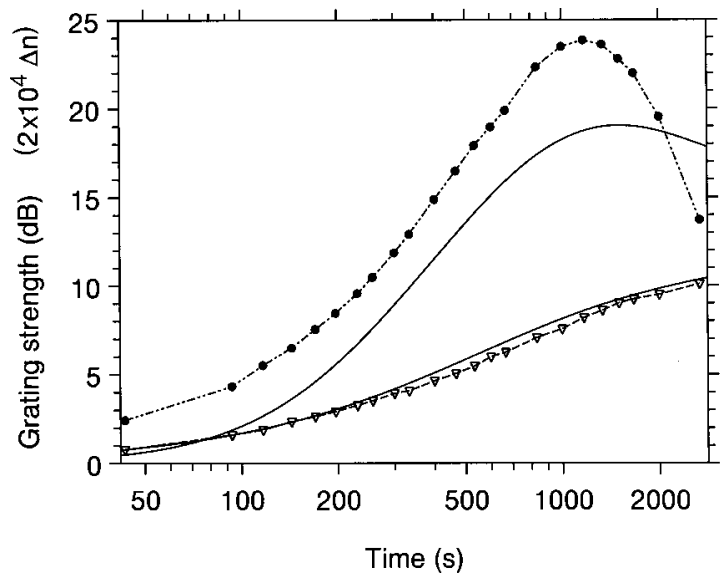

FIG. 4. Comparison of model prediction and experiment for an exposure not included in the model optimization. The experimental parameters are $18 \% \mathrm{Ge}, \lambda=248 \mathrm{~nm}, 20-\mathrm{Hz}$ repetition rate, $I_{\text {laser }}$ $=300 \mathrm{~mJ} / \mathrm{cm}^{2}$ and $L_{\text {exp }}=5 \mathrm{~mm}$. The solid circles connected with a dash-dotted line are the measured grating strengths in transmission $(\mathrm{dB})$ and the open triangles connected with a dashed line are the measured changes in effective refractive index (multiplied by 2 $\times 10^{4}$ ). The solid lines are the predictions from the model. The agreement with the effective index change is excellent, but the agreement with the absolute grating strength is imperfect. However, the qualitative shape is correct.

space the $c^{3} \Pi_{u}^{+} \mathrm{H}_{2}$ molecules are predissociated by the repulsive $b$ state and the remaining $c$-state molecules decay very slowly through a forbidden transition. ${ }^{59}$ It is of course also necessary to correct for the glass absorption and some geometrical factors when comparing this prediction with experiments. Another related prediction is that after very long exposure time for loaded glass a significant fraction of the hydrogen will have undergone dissociation due to the boundfree triplet transition. Part of the energy of the fragments will almost certainly go into chemical reactions and result in damage to the glass structure. Most likely there will be significant structural changes and high concentration of $\mathrm{OH}$ groups and therefore strong scattering and absorption in the glass. In this limit the model may not describe the effects accurately. The same limit will probably not be reached for unloaded glass except when very high UV intensities are used, whereby the glass is directly damaged. Another completely unrelated prediction is that the material dispersion for the UV induced part of the refractive index will be much higher than for the intrinsic part of the refractive index due to the closer proximity of the UV-absorption for the defects.

\section{DISCUSSION AND CONCLUSION}

A dipole-quadrupole model has been presented for UVinduced processes in germanium-doped silica glass, including the effects of hydrogen or deuterium loading. The model is intrinsically of microscopic nature, but it should be emphasized that this does not rule out that part of the effect can be a compaction of the glass, since defects may well work to increase the density. The model assumes that germanium atoms work as gates for transferring energy into the material.
By making plausible assumptions about the transfer mechanisms it has been possible to give a quantitative description of a very large number of UV exposure experiments. The model assumes that two types of defects are formed. The absolute energies, thermal activation energies, and rates for both these defects are determined. Their contributions to the index change are estimated. However, an absolute determination of these contributions must await independent measurements of the absolute defect concentrations. This may, for instance, be possible using ${ }^{29} \mathrm{Si}$ NMR spectroscopy.

Generally, good agreement is found with experimental data, thereby justifying the basic assumptions of the model. It is able to describe results of UV exposures using wavelengths within the range of the 242-nm absorption, at least one order of magnitude variation of the germanium concentration, most experimentally accessible hydrogen or deuterium concentrations, and more than ten orders of magnitude variation in the UV intensity. Furthermore, it describes the refractive index change including the properties of UVwritten gratings, the change in absorption, and the UVinduced visible luminescence. Finally, it gives a good description of accelerated aging experiments and normal thermal decay, and it is therefore able to predict the longtime stability of UV-written gratings. For this purpose Eq. (3) reduces to the following simple expression:

$$
\begin{gathered}
c_{1}=c_{3}=0, \\
c_{2}=k_{x}\left(\frac{r_{1}}{r}\right)^{10} \exp \left(-\frac{\Delta E_{x}}{R T}\right), \\
c_{4}=k_{y}\left(\frac{r_{1}}{r}\right)^{10} \exp \left(-\frac{\Delta E_{y}}{R T}\right) .
\end{gathered}
$$

A more detailed treatment of the thermal properties will be given in a later publication.

The most significant achievement is that the model uses one universal parameter set to describe all the results in a self-consistent way. Another very important result is that it can explain the complicated structure of the red luminescence spectra and relate this to the fundamental structure of the glass determined from x-ray spectroscopy. Finally, it gives a more reasonable explanation for the thermal decay of UV-induced defects compared to conventional methods. Instead of assuming an unreasonably broad spectrum for the thermal activation energies, it relates the behavior to the radial dependence of the transition probabilities and the existence of two competing defects with opposite influence on the refractive index.

The model is able to make several new predictions of both detailed UV-writing experiments and of qualitatively new effects. This paper gives a few examples of different types of predictions to serve as illustration and as tools which can be used to corroborate or reject the model based on new experimental results.

The model may also be used to make a more general definition of the term photosensitivity. One simple possibility could be to define the photosensitivity as the initial slope of the index change curve which is proportional to the $c_{1}$ pa- 
rameter according to Eq. (1). Within the validity range of the model this would give only three ways to increase the photosensitivity in good agreement with intuition. The first one is to increase the germanium concentration which will decrease the average size of $r$ and give rise to a nonlinear increase of the photosensitivity. Another possibility is to increase the UV intensity which will give rise to second-order terms proportional to $\alpha_{2} I_{\text {laser }}^{2}$. Finally, loading with hydrogen or deuterium can be used. This increases $c_{1}$ in several ways. It should be emphasized that this definition is not the only possibility within the model and that beyond the validity range of the model many other possibilities exist for definitions and ways to get an increase. The most prominent increases beyond those described by the model have been obtained by doping with other materials or by oxygendeficient glass deposition. In the future it will be natural to extend the model to take some of these effects as well as the thermal activation of hydrogen and deuterium in the glass into account.

In conclusion, the UV dipole-quadrupole model presented here is a very useful tool for both physicists and chemists studying UV-induced processes and for engineers who would like to predict the performance of a new type of fiber or waveguide. The free parameters in the model have been determined by simple adaptation (without curve fitting) to a few spectra covering a large parameter range. Despite this simple procedure, the model gives good, quantitative predic- tions in all cases where it has been tried. If one should later choose to perform curve fitting of the model to a selected data set within a narrow parameter range, it is likely to be a valuable tool delivering precise and physically reasonable approximations to experimental spectra. In this way, extrapolations to nearby regimes will still be accurate, which may be important for production purposes.

The most severe argument against the model is that it contains many free parameters. This is typical for a detailed description of a complicated amorphous material such as glass. The many parameters are also to a very large degree resulting from the need to describe many different types of effects, each demanding a few parameters (e.g., for normalization). Of course it would be preferable if most of the free parameters could be determined from first principles. At the moment this is not possible, but for several of the parameters such as branching ratios, absolute energy levels, and the activation energies, there is good reason to expect that they can be determined from quantum chemical calculations in the near future.

\section{ACKNOWLEDGMENTS}

I would like to thank A.N. Neergaards og Hustrus Fond for financial support and Jesper Lægsgaard for fruitful discussions.
${ }^{1}$ K. O. Hill, Y. Fujii, D. C. Johnson, and B. S. Kawasaki, Appl. Phys. Lett. 32, 647 (1978).

${ }^{2}$ G. Meltz, W. W. Morey, and W. H. Glenn, Opt. Lett. 14, 823 (1989).

${ }^{3}$ H. Hosono, D. L. K. Y. Abe, R. A. Weeks, K. Muta, and H. Kawazoe, Phys. Rev. B 46, 11445 (1992).

${ }^{4}$ M. Fujimaki, T. Watanabe, T. Katoh, T. Kasahara, N. Miyazaki, and Y. Ohki, Phys. Rev. B 57, 3920 (1998).

${ }^{5}$ L. Skuja, J. Non-Cryst. Solids 239, 16 (1998).

${ }^{6}$ J. Nishii, K. Kintaka, H. Hosono, H. Kawazoe, M. Kato, and K. Muta, Phys. Rev. B 60, 7166 (1999).

${ }^{7}$ L. Skuja, M. Hirano, and H. Hosono, Phys. Rev. Lett. 84, 302 (2000).

${ }^{8}$ M. G. Sceats, G. R. Atkins, and S. B. Poole, Annu. Rev. Mater. Sci. 23, 381 (1993).

${ }^{9}$ H. G. Limberger, P. Y. Fonjallaz, R. P. Salathé, and F. Cochet, Appl. Phys. Lett. 68, 3069 (1996).

${ }^{10}$ M. Douay, W. X. Xie, T. Taunay, P. Bernage, P. Niay, P. Cordier, B. Poumellec, L. Dong, J. F. Bayon, H. Poignant, and E. Delevaque, J. Lightwave Technol. 15, 1329 (1997).

${ }^{11}$ F. Piao, W. G. Oldham, and E. E. Haller, J. Non-Cryst. Solids 276, 61 (2000).

${ }^{12}$ T. Erdogan, V. Mizrahi, P. J. Lemaire, and D. Monroe, J. Appl. Phys. 76, 73 (1994).

${ }^{13}$ J. Rathje, M. Kristensen, and J. E. Pedersen, J. Appl. Phys. 88, 1050 (2000).

${ }^{14}$ S. Hüfner, Optical Spectra of Transparent Rare Earth Compounds (Academic Press, New York, 1978).
${ }^{15}$ R. Reisfeld and C. K. Jørgensen, Lasers and Excited States of Rare Earths (Springer, Berlin, 1977).

${ }^{16}$ V. B. Sulimov, V. O. Sokolov, E. M. Dianov, and B. Poumellec, Phys. Status Solidi A 158, 155 (1996).

${ }^{17}$ M. J. Yuen, Appl. Opt. 21, 136 (1982).

${ }^{18}$ G. Pacchioni and R. Ferrario, Phys. Rev. B 58, 6090 (1998).

${ }^{19}$ K. O. Hill, B. Malo, F. Bilodeau, D. C. Johnson, and J. Albert, Appl. Phys. Lett. 62, 1035 (1993).

${ }^{20}$ M. Svalgaard, C. V. Poulsen, A. Bjarklev, and O. Poulsen, Electron. Lett. 30, 1401 (1994).

${ }^{21}$ R. Kashyap, Fiber Bragg Gratings (Academic Press, San Diego, 1999).

${ }^{22}$ J. Albert, B. Malo, D. C. Johnson, and K. O. Hill, Opt. Lett. 19, 387 (1994).

${ }^{23}$ M. Sejka, P. Varming, J. Hübner, and M. Kristensen, Electron. Lett. 31, 1445 (1995).

${ }^{24}$ E. M. Dianov, D. S. Stardubov, S. A. Vasiliev, A. A. Frolov, and O. I. Medvedkov, Opt. Lett. 22, 221 (1997).

${ }^{25}$ V. M. Marchenko, Glass Phys. Chem. 21, 263 (1995).

${ }^{26}$ M. Fujimaki, T. Kasahara, S. Shimoto, N. Miyazaki, S. Tokuhiro, K. S. Seol, and Y. Ohki, Phys. Rev. B 60, 4682 (1999).

${ }^{27}$ J. Hübner and M. Kristensen (unpublished).

${ }^{28}$ E. M. Dianov, V. O. Sokolov, and V. B. Sulimov, Quantum Electron. 27, 600 (1997).

${ }^{29}$ E. M. Dianov, D. S. Starodubov, and A. A. Frolov, Electron. Lett. 32, 246 (1996).

${ }^{30}$ F. Goutaland, H. Kuswanto, A. Yahya, A. Boukenter, and Y. Ouerdane, Philos. Mag. B 79, 2137 (1999). 
${ }^{31}$ J. Canning, D. Moss, M. Åslund, and M. Bazylenko, Electron. Lett. 34, 366 (1998).

${ }^{32}$ M. Svalgaard, Ph.D. thesis, Mikrolektronik Centret, DTU, Lyngby, 1997.

${ }^{33}$ J. Canning and M. G. Sceats, Opt. Lett. 19, 1119 (1994).

${ }^{34}$ R. Tohmon, Y. Shimogaichi, S. Munekuni, Y. Ohki, and Y. Hama, Appl. Phys. Lett. 54, 1650 (1989).

${ }^{35}$ T. Förster, Ann. Phys. 2, 55 (1948).

${ }^{36}$ D. L. Dexter, J. Chem. Phys. 21, 836 (1953).

${ }^{37}$ T. T. Basiev, V. A. Malsyshev, and A. K. Przhevuskii, in Spectroscopy of Solids Containing Rare Earth Ions, edited by A. A. Kaplyanskii and R. M. Macfarlane (Elsevier Science, New York, 1987), Chap. 6.

${ }^{38}$ I. I. Sobelman, Atomic Spectra and Radiative Transitions (Springer, Berlin, 1979).

${ }^{39}$ D. R. Yarkony, J. Chem. Phys. 90, 7164 (1989).

${ }^{40}$ O. Vahtras, H. Ågren, P. Jørgensen, H. J. A. Jensen, T. Helgaker, and J. Olsen, J. Chem. Phys. 96, 2118 (1992).

${ }^{41}$ P. Jørgensen (private communication).

${ }^{42}$ M. G. Sceats (private communication).

${ }^{43}$ J. A. Leegwater and S. Mukamel, Phys. Rev. A 49, 146 (1994).

${ }^{44}$ P. J. Lemaire, R. M. Atkins, V. Mizrahi, and W. A. Reed, Electron. Lett. 29, 1191 (1993).

${ }^{45}$ B. E. Warren, J. Am. Ceram. Soc. 17, 249 (1934).

${ }^{46}$ R. L. Mozzi and B. E. Warren, J. Appl. Crystallogr. 2, 164 (1969).
47 M. Svalgaard, OSA Tech. Digest Series 22, 160 (1995).

${ }^{48}$ R. M. Atkins and V. Mizrahi, Electron. Lett. 28, 1743 (1992).

${ }^{49}$ M. Gallagher and U. Österberg, Appl. Phys. Lett. 63, 2987 (1993).

${ }^{50}$ M. Poirier, S. Thibault, J. Lauzon, and F. Ouellette, Opt. Lett. 18, 870 (1993).

${ }^{51}$ D. Marcuse, Bell Syst. Tech. J. 56, 703 (1977).

${ }^{52}$ L. Riseberg and M. Weber, in Progress in Optics, edited by E. Wolf (North-Holland, Amsterdam, 1976), Vol. XIV.

${ }^{53}$ W. H. Press, B. P. Flannery, S. A. Teukolsky, and W. T. Vetterling, Numerical Recipes (Cambridge University Press, Cambridge, England, 1989).

${ }^{54}$ G. Meltz and W. W. Morey, Proc. SPIE 1516, 185 (1991).

${ }^{55}$ J. Lauzon, M. G. Sceats, P. A. Krug, and F. Ouellette, Technical Digest of OFC ' 94 (Optical Society of America, Washington, D.C., 1994), p. 137.

${ }^{56}$ H. Scher, M. F. Shlesinger, and J. T. Bendler, Phys. Today 44 (1), 26 (1991).

${ }^{57}$ J. Hübner, Ph.D. thesis, Mikrolektronik Centret, DTU, Lyngby, 1998.

${ }^{58}$ F. Bilodeau, B. Malo, J. Albert, D. C. Johnson, and K. O. Hill, Opt. Lett. 18, 953 (1993).

${ }^{59}$ W. Koot, W. J. van der Zande, P. H. P. Post, and J. Los, J. Chem. Phys. 90, 4826 (1989). 